\title{
NÉOLOGIE, NÉONYMIE ET DICTIONNAIRES
}

\author{
Jean-François SABLAYRolLES \\ Université Sorbonne Paris Cité - HTL UMR 7597
}

\begin{abstract}
New words are added to dictionaries because they cease being neologisms, sometimes long time before that. The trivialization (terms from specialized fields becoming part of the everyday language), the exchange of terms between various domains, semantic neology and the existence of homonymous creations, multiple creations and renominations turn into problems which are often difficult to solve for the lexicographers.
\end{abstract}

Pour ma collègue Alicja Kacprzak, en hommage à ses travaux érudits et en remerciement de notre collaboration de près d'une dizaine d'années.

\section{INTRODUCTION}

Les dictionnaires n'entrent les néologismes qu'après qu'ils ont perdu ce statut, et parfois longtemps après, comme des recherches de Camille Martinez (2013 ; Elchakar, Martinez, 2008) l'ont bien montré. J'ai aussi mis en évidence la faible proportion de mots datés de moins de dix ans dans les nouvelles entrées dans la nomenclature du Petit Robert (Sablayrolles, 2011). Il y a en effet dans le millésime 2008 à peine plus de 70 lexies dont la date de première attestation se situe entre 1998 et 2007, mais certaines ont été entrées l'année même de la date de leur première attestation (deux : customisaton et mot-rébus dans le millésime 2001) ${ }^{1}$ ou peu d'années après. Ces quelques dizaines de lexies récentes représentent peu par rapport aux centaines qu'il devrait y avoir si toutes les lexies entrées dans la

${ }^{1}$ Il faut tenir compte du décalage entre le millésime affiché et la date de parution réelle, en juin de l'année précédente, ainsi que du temps d'élaboration et de fabrication de chaque nouveau millésime. 
nomenclature avaient moins de dix ans. Avec l'hypothèse d'une moyenne d'une centaine de nouvelles entrées chaque année, si toutes dataient de moins de dix ans, on en aurait cinq cents, pour les dix années 1998-2007 (100 x (10:2)).

Il faut donc renverser la doxa : ce n'est pas parce qu'un mot est absent du dictionnaire qu'il est néologique mais c'est parce qu'il a perdu ce statut qu'il entre dans un ou des dictionnaire(s), qu'il est "dictionnarisé » ou " lexicographié », si je peux me permettre ces néonymes bien utiles pour ne pas confondre avec lexicalisé $e^{2}$. Ces retards de « lexicographisation » valent autant pour les néonymes (termes nouveaux des domaines spécialisés) que pour les néologismes de la langue générale, avec des situations fort différentes selon les cas. On constate par ailleurs, avec des néologismes d'emploi, des échanges entre domaines spécialisés aussi bien qu'entre domaines spécialisés et langue générale, et ce dans les deux sens, sans qu'ils soient toujours explicités dans les dictionnaires.

\section{BANALiSATION, RENOMINATION ET SYNONYMES POPUlaiRES}

L'utilisation du dictionnaire comme corpus d'exclusion pour le repérage des néologismes ne peut être automatique et doit prendre en compte le fait que les dictionnaires généraux sont incomplets, comme le reconnaissent d'ailleurs les lexicographes dans leur préface ou introduction. Parmi leurs lacunes figurent précisément des termes, considérés comme spécialisés, tant qu'ils ne se sont pas diffusés dans la langue générale. On observe aussi dans la langue des renominations et des synonymes populaires, présents ou absents des dictionnaires selon les cas.

\subsection{La banaLisation}

La recherche traditionnelle de la date de première attestation occulte le fait qu'une unité lexicale peut être plusieurs fois nouvelle ${ }^{3}$, une fois quand elle est créée comme terme (c'est un néonyme) et une autre fois quand elle entre dans

\footnotetext{
${ }^{2}$ Dictionnariser est présenté comme un néologisme par le dictionnaire en ligne Wiktionnaire, consulté en mars 2018. Lexicographier se trouve assez souvent dans des articles de linguistes quand on regarde les pages indiquées dans une requête sur Google.

${ }^{3} \mathrm{D}$ 'autres cas du caractère répétitif de la nouveauté existent qui ne nous retiendront pas ici : les créations multiples simultanées ou étalées dans le temps (Ségolène Royal ignorait que des créateurs d'un jeu sur internet avaient créé le mot bravitude avant elle, comme le montre Arrivé, 2009), la réintroduction dans la langue de mots qui avaient existé puis qui avaient disparu (v. Sablayrolles, 2000 : 191-193; 2010), etc. Il en va de même de la mésaventure du publicitaire qui a créé le néologisme anatopisme accompagné de sa définition, sur le mode lexicographique, "agréable sensation de dépaysement ressenti par des vacanciers... » sur fond de paysage idyllique, alors qu'anatopisme existe comme terme spécialisé de psychiatrie pour dénommer les maux des personnes déracinées.
} 
la "langue commune », avec un statut de néologisme, comme ce fut le cas de biométrisation, hub, nanocapteur, etc. C'est le phénomène de la banalisation que Galisson (1978) avait bien mis en évidence et bien décrit : nous ne nous $\mathrm{y}$ attarderons donc pas si ce n'est pour dire que ce changement de statut est une innovation qui relève de la néologie, sans doute pas cependant dans son aspect prototypique. Mais pour un membre de la communauté linguistique non spécialiste d'un domaine la rencontre d'un terme dans des énoncés de langue générale fait figure de néologisme. Ce phénomène de banalisation est une des explications du décalage temporel entre la date de première attestation indiquée et la date d'entrée dans le dictionnaire.

Par ailleurs, ce sont souvent des circonstances particulières qui font entrer dans la langue courante des termes. Ainsi les fadettes (factures détaillées) du domaine des télécommunications lors de l'affaire Bettencourt (un juge se faisant communiquer les fadettes d'un journaliste du Monde pour découvrir ses sources). Là encore on constate un retard d'enregistrement dans la nomenclature des dictionnaires. Ce mot est entré dans le millésime 2014 du Petit Robert (qui date le mot de 1999 avec un exemple du Monde de cette même année). Il l'avait été l'année précédente dans le Petit Larousse. Wiktionnary le donne comme un néologisme et fournit un exemple de 2010.

De la même manière ce sont les événements particuliers qu'ont été de grandes catastrophes écologiques occasionnées par des naufrages de cargos qui ont conduit à faire entrer ces dernières décennies dans la langue courante des distinctions faites auparavant uniquement par les spécialistes du domaine maritime entre charbonnier, chimiquier, méthanier, minéralier, pétrolier, porte-conteneurs, vraquier $^{4}$, etc. en fonction des marchandises transportées et des différents types de pollution produits.

Faisons une ultime remarque sur ce point de la banalisation et de la lexicographisation. On peut s'interroger sur la cohérence des indications de domaine (celles du Petit Robert reproduites dans la note précédente pour les différents types de cargos laissent planer un doute à ce sujet puisqu'il n'y en a pas pour trois types de cargos, que deux sont étiquetés TECHN. et un MAR.) ainsi que sur leur statut dans les dictionnaires. Cela veut-il dire que ces termes sont cantonnés à ces domaines spécialisés ? Mais alors pourquoi figurent-ils dans le dictionnaire de langue générale ? Ou bien cela veut-il dire que c'est leur origine et

${ }^{4}$ À l'entrée cargo du $P R$ on trouve charbonnier (pas de date pour cette acception ni indication de domaine), chimiquier (1995, sans marque), minéralier (1960, sans marque), vraquier (1973, avec marque MAR.) ainsi que cargo pétrolier, mais pas porte-conteneurs (1972, remplaçant portecontainers de 1967) ni méthanier (milieu du $\mathrm{XX}^{\mathrm{e}} \mathrm{s}$ ) qui ont pourtant des entrées et qui sont précédés de l'indication de domaine TECHN (et pas MAR.). 
leur emploi le plus courant, qu'un honnête homme contemporain peut rencontrer - ce qui justifie leur présence dans la nomenclature - mais qu'il doit se garder d'employer dans un certain type d'énoncés où seule la langue courante et/ou académique est admise (une dissertation, un discours épidéictique par exemple) ? Sur cinq dictionnaires monovolumaires contemporains, trois ne donnent aucune information précise à ce sujet, se contentant de dresser une liste des abréviations des spécialités (Dictionnaire Hachette, 2009, p. 11), des rubriques (Petit Larousse, 2008, p. XXXIV) ou même une table indistincte de toutes les abréviations dans Nouveau Littré, 2006, p. 15-17. En revanche les lexicographes du Petit Robert et de Lexis donnent des informations intéressantes à ce sujet. Une remarque de Petit Robert (2010 : XXXI) en tête du «tableau des termes, signes conventionnels et abréviations du dictionnaire » indique que :

Dans ce tableau terme présente un mot de spécialistes qui fait partie d'une terminologie (technique, didactique, scientifique) et non du vocabulaire courant. Les noms des domaines ne renvoient pas à des domaines encyclopédiques, ils indiquent que le terme dont ils précèdent la définition appartient au vocabulaire des spécialistes de ce domaine.

Un paragraphe de la préface du Lexis (1992 : VII) est consacré au « Vocabulaire des sciences et des techniques » distinct du « vocabulaire courant» ainsi que des « locutions et syntagmes figés »:

Les mots et les sens appartenant à un domaine technique ou scientifique précis sont signalés par une abréviation ou « rubrique », dont on trouvera la liste complète en tête d'ouvrage p. XIII. Cette liste, établie d'après la classification mécanographique du Grand Larousse encyclopédique, montre qu'aucun domaine de la connaissance n'a été négligé. Cependant, les différentes sciences et techniques n'ont pas donné lieu à un traitement similaire : le contemporain a été privilégié par rapport à l'ancien. En ce qui concerne les vocabulaires scientifiques, on a fait une distinction entre les mots qui permettent de décrire le fonctionnement de la science et les mots qui appartiennent à des énumérations ouvertes : on ne pouvait raisonnablement envisager, dans le cadre d'un seul volume, de recenser, en botanique et en zoologie, les innombrables animaux et plantes exotiques, en géologie les noms de tous les minéraux, roches et fossiles.

La philosophie des deux dictionnaires qui s'expriment sur ce sujet n'est donc pas la même puisque Lexis se réclame, sur cet aspect, d'une conception encyclopédique que récuse pour sa part le Petit Robert. En revanche le choix des termes retenus dans ce dernier n'est pas explicité. Ce qui est sûr c'est qu'ils sont 
présentés comme ne relevant pas de la langue commune, avec les conséquences que cela peut avoir pour leur emploi par les utilisateurs du dictionnaire. On peut raisonnablement faire l'hypothèse que l'insertion de ces termes vise plus la lecture de textes que leur production.

\subsection{LA RENOMINATION}

La banalisation peut avoir des conséquences dans le domaine spécialisé d'origine : des mots spécialisés entrés dans la langue courante deviennent inadéquats aux yeux des spécialistes du domaine qui les remplacent dans le domaine spécialisé. Ce fut le cas de atomique auquel les physiciens ont substitué nucléaire dans un certain nombre d'emplois. La fission de l'atome est utilisée dans des centrales nucléaires plus que dans des centrales atomiques pour produire de l'énergie d'origine nucléaire, plus que d'origine atomique. Quant aux écologistes, ils veulent diminuer le poids du nucléaire et pas de l'atomique. Ces cas de renomination d'un concept par un autre terme du fait de la banalisation du premier terme sont rares comparativement à ceux qui se produisent lorsque des progrès techniques conduisent à opérer des distinctions entre des objets anciens et des nouveaux, qu'ils aient le même usage (disque noir ou vinyle vs disque compact / $C D$, ou encore appareil argentique vs appareil numérique à la place de l'ancien appareil photo, fixe remplaçant téléphone depuis la multiplication des portables ${ }^{5}$ ) ou qu'ils aient des usages différents (machine à laver devenant lave-linge avec l'apparition et la diffusion des lave-vaisselle).

\subsection{LES SYNONYMES POPULAIRES}

Inversement, des dénominations populaires qui doublent des termes peuvent tarder à entrer dans la nomenclature de dictionnaires, voire en être (définitivement ?) exclues. Les agents de régulation des flux dans le domaine des transports en commun ont immédiatement été surnommés pousseurs par les usagers et on ne peut que constater l'absence de ce pousseur dans $P L, P R$ et deux autres dictionnaires monovolumaires millésimés.

Dans les échanges courants de la vie quotidienne, on note l'abondance de mots vagues pour désigner des objets que l'on ne sait pas nommer ou dont on ne veut pas, pour des raisons diverses, utiliser la dénomination propre, avec le recours

${ }^{5}$ Notons l'ambiguïté de ce mot qui dénomme aussi bien un téléphone qu'un ordinateur. 
à chose, machin, truc, bitoniau, etc. (v. Gérard Petit, 2009). La préparation de cette contribution m'a rappelé à ce sujet la chanson humoristique des Frères Jacques à propos de la ceinture de sécurité où aucun des éléments en jeu n'est nommé par un terme propre mais est évoqué par ces mots sans véritable signifié 6 . Or, la recherche dans des dictionnaires courants et des encyclopédies en ligne montre qu'ils ne proposent aucune description des différents éléments d'un dispositif d'une ceinture de sécurité et aucune dénomination de ses éléments constitutifs. Alors qu'au mot ceinture (en général) sont indiqués trois modes de fermeture (boucle, nœud, mécanisme de serrage), il est remarquable que le personnel de cabine dans les avions parle au mieux ${ }^{7}$ de boucle alors que la partie qui reçoit l'embout (si ce mot convient bien) ne ressemble en rien à une boucle ${ }^{8}$. Interrogés à ce sujet des ingénieurs aéronautiques ne connaissaient pas non plus les termes spécialisés, qui doivent pourtant bien exister et être employés par les sous-traitants fabriquant les sièges et ces dispositifs de sécurité (avec de surcroît des noms pour chacune des pièces composant ces objets).

Notons encore au sujet des synonymes populaires que l'existence de mots de la langue générale (contusion daté de 1314 ou meurtrissure daté de 1535) ou de termes d'origine scientifique (ecchymose daté de 1540, hématome apparu en 1855) n'empêche pas l'apparition postérieure de mots imagés dans la langue courante pour dénommer un même type de réalité : un bleu (attesté en 1863).

${ }^{6}$ Voici un extrait de la chanson, où l'on remarque que truc et machin s'emploient alternativement pour les parties mâle et femelle du dispositif et aussi pour le système d'accrochage :

$\mathrm{Y}$ a un bout avec un truc

$\mathrm{Y}$ a un bout avec un machin

Quand on enfonce le bout qu'a l'truc

Il doit faire clic dans le machin

L'bout qui s'termine par un machin

S'accroche à gauche sur un truc

Et comme on ne trouve jamais l'truc

On laisse pendouiller le machin

Seul'ment le bout qui a un truc

Lui s'accroche pas sur un machin

C'qui fait que lorsqu'on cherche le truc

Il est paumé sous les coussins.

${ }^{7}$ La stratégie d'évitement de l'emploi des termes propres se fait par le recours aux gestes du démonstrateur ou aux dessins de la plaquette des consignes de sécurité, avec le recours à un déictique : "vous ajustez et bouclez votre ceinture en faisant comme ça » avec le geste et ou le dessin.

${ }^{8}$ On dit régulièrement aussi boucler sa ceinture. On observe dans ces emplois de boucle et de boucler des extensions d'emploi pour des mécanismes ayant la même fonction que les boucles de ceinture mais qui n'ont plus la forme d'anneau qui a donné son nom à cet élément de fermeture. 
Il ne faut pas confondre ces emplois de mots familiers ou de mots vagues avec la persistance de l'utilisation de noms anciens de la langue courante alors que des termes scientifiques spécifiques sont apparus ultérieurement. Le mot saignement est daté de 1680 et on continue de dire saignement de nez que n'a pas détrôné le terme médical plus tardif épistaxis apparu en 1795 par exemple. Depuis au moins le XII ${ }^{\mathrm{e}}$ siècle on dit couramment jaunisse alors que sont apparus depuis, en 1578, l'emprunt savant ictère « maladie avec coloration jaune de la peau » puis " coloration jaune de la peau sans autre symptôme de maladie " depuis 1902, en 1660, hépatite " affection inflammatoire du foie ", en 1859, cholémie " passage d'éléments de bile dans le sang ». Seul le recours à des dictionnaires retraçant l'histoire des mots permet de faire le départ entre les synonymes populaires qui apparaissent après des termes (cas de pousseur et de bleu) et ceux qui continuent d'exister après l'apparition de termes (cas de saignement de nez et jaunisse).

\section{NOUVEAUX EMPLOIS}

À côté des néologismes formels seuls envisagés jusque-là, il y a aussi des innovations d'emploi (sémantico-syntaxiques). Quelle que soit la macrostructure adoptée - les lexicographes des dictionnaires généraux commercialisés montrent, sauf l'exception qu'est Lexis, une prédilection pour une approche polysémique des unités de préférence au dégroupement en plusieurs entrées -, on peut souvent regretter l'absence d'indication de la date des nouvelles acceptions (ou des nouveaux homonymes) avec des extensions à d'autres domaines ou des échanges entre un domaine spécialisé et la langue générale, ainsi que l'absence d'indication $\mathrm{du}$ lien logique qui unit l'emploi originel et l'emploi nouveau.

\section{1. ÉCHANGES ENTRE DOMAINES DE SPÉCIALITÉ}

Les dictionnaires n'explicitent en effet pas toujours les échanges de termes entre domaines de spécialités proches ou éloignés, comme le passage d'un terme d'un sport à un autre, pour des termes qui ont été néologiques mais qui ne le sont plus à l'heure actuelle (v. Sablayrolles, 2005). On remarque d'ailleurs que les dictionnaires n'indiquent qu'imparfaitement les différents domaines dans lesquels ces termes sont utilisés (dribbler) quand ils ne les omettent pas complètement (remiser). 
- sports collectifs de ballon : dribbler (foot $1895 \rightarrow$ basket $1951 \rightarrow$ handball 1963)

Seuls le foot et le basket, mis sur le même plan, sont présents dans le $P R$. Le passage du premier sport au second n'est pas noté et le troisième est absent. Le $P L$ n'indique aucune date et aucune filiation mais donne un quatrième sport, le hockey. Hachette et Nouveau Littré ne donnent le nom d'aucun sport mais leurs définitions, avec les mots balle et ballon, excluent le hockey.

- chasse $\rightarrow$ équitation : ramingue du « faucon volant de branche en branche » au « cheval rétif»

Les deux acceptions sont présentes dans le $P R$, sans explicitation de leur lien. En revanche Hachette et Nouveau Littré ne donnent que la seconde acception. Le mot est absent du $P L$.

- escrime $\rightarrow$ boxe : remiser « riposter par un coup droit sans reprise de garde » Ce mot n'est présent dans un dictionnaire de l'escrime sur internet ${ }^{9}$ que sous la forme nominale. On le trouve dans un glossaire de la box $\mathrm{e}^{10}$ aux articles opportunité et riposte. En revanche il est absent, tant comme nom que comme verbe, dans les dictionnaires contemporains d'usage courant.

- escrime $\rightarrow$ autres sports dont football et rugby : feinter (intransitif originellement)

Le $P R$ donne un premier emploi intransitif (1859) avec un exemple d'escrime puis un deuxième emploi, transitif (1931) dans le domaine sportif, avec le foot et le rugby (et une acception familière « tromper »). Hachette ne donne pour l'acception sportive que la construction intransitive, sans mention de sports particuliers ni exemples (l'autre emploi est figuré « tromper »). Dans le domaine sportif Nouveau Littré donne d'abord la construction transitive avec l'exemple de feinter le gardien de but. La seconde acception « tromper » est donnée comme familière et il y en a une troisième, en emploi intransitif, pour la seule escrime. $P L$ a une présentation analogue.

- cyclisme $\rightarrow$ athlétisme (1930) : changer de braquet « accélérer »

Dans trois dictionnaires, $P R$, Hachette et Nouveau Littré, l'expression figure sous l'entrée braquet, terme de cyclisme, mais avec un sens général « changer de rythme » sauf Hachette qui donne bizarrement « changer d'attitude, d'orientation », mais des pages trouvées avec Google confirment cet emploi. L'expression est

\footnotetext{
${ }^{9} \mathrm{http}: / /$ www.synec-doc.be/escrime/dico/dico_escrime-R.html\#Heading451 (dernière consultation : le 02.12.2017).

${ }^{10} \mathrm{http}: / /$ fr.wikipedia.org/wiki/Glossaire_de_la_boxe\#R (dernière consultation : le 02.12.2017).
} 
absente du $P L$. Un dictionnaire du sport sur internet ${ }^{11}$ indique : "Par extension, on a parlé de braquet dans d'autres sports faisant appel à des changements de rythme, de vitesse. 1 . En athlétisme où, depuis 1930, changer de braquet signifie " accélérer », 2. au figuré, comme au rugby, il s'agit de se remettre en question, redoubler d'efforts ». Wiktionnaire ne donne que " passer à la vitesse supérieure » et fait appel aux contributeurs pour fournir une étymologie manquante.

- nautisme $\rightarrow$ ski : godille et godiller

$P R$ donne pour ce mot daté de 1792 les deux emplois l'un après l'autre, mais sans date pour le second. PL, Nouveau Littré et Hachette donnent ces deux mêmes emplois sans date.

- nautisme $\rightarrow$ cyclisme : enlevage

Seul l'emploi pour les sports nautiques est indiqué dans $P R$, qui le date de 1874. Hachette et Nouveau Littré donnent ce même emploi, sans date. L'emploi sportif de ce mot est absent du $P L$.

- hippisme (turf) $\rightarrow$ autres sports : handicap « désavantage imposé à un concurrent pour rétablir l'égalité des chances »

Seul $P R$ indique que l'emploi hippique a précédé l'extension aux autres sports. Les autres dictionnaires mettent tout sur le même plan. Cette acception sportive est mise en première place dans tous les dictionnaires sauf $P L$ qui met « désavantage, infirmité ou déficience » en tête d'article.

Les exemples cités montrent que ce mécanisme d'échange n'est pas un procédé nouveau mais un mécanisme actionné à toute époque. Il s'applique à des domaines proches ou plus lointains comme récemment le vocabulaire de la médecine en informatique avec ver, virus, infecter, etc. Plus remarquables sont les transferts d'un domaine spécialisé à la langue générale et vice-versa.

\subsection{INNOVATION D'EMPLOIS SYNTACTICO-SÉMANTIQUES}

Il ne faut pas confondre les nouveaux emplois de termes dans la langue générale avec la banalisation vue précédemment, où l'unité garde son signifié inchangé. Il s'agit maintenant de relever des innovations syntactico-sémantiques liées au changement de registre (nous retenons ici la distinction opérée par Joëlle GardesTamine (1990: 103) entre registre et niveau).

\footnotetext{
${ }^{11}$ books.google.fr/books?isbn=2354253532 (dernière consultation : le 02.12 .2017$)$.
} 


\subsubsection{D'Un DOMAINE SPÉCIALISÉ À LA LANGUE GÉNÉRALE}

Sont souvent indiqués, avec la mention fig., les passages de termes dans la langue générale, comme $K O$, carton jaune, carton rouge qui viennent du domaine sportif, ou, pour des termes issus du domaine de la transmission des informations : être câblé, branché ${ }^{12}$, etc. Il s'agit dans ces cas de domaines spécialisés auxquels a accès une large frange de la population. On peut s'interroger sur le type de mécanisme à l'origine du nouvel emploi, avec deux types de solution : soit il s'agit d'emplois figurés volontaires, par métonymie, métaphore ou autres figures (v. Gévaudan, Koch, 2010), soit il s'agit d'extension ou de restriction d'emplois qui interviennent entre un locuteur et ses récepteurs qui interprètent le mot avec un sens plus large ou plus étroit que le sens encodé et qui le réemploient avec ce nouveau sens. Dans ces derniers cas, il n'y a pas à proprement parler néologie (v. Nyckees, dans la lignée de Bréal et surtout de Meillet qui met l'accent sur la « discontinuité de la transmission du langage » (avec des contextes de transition), sur les emprunts sociaux et l'utilisation d'un mot avec le sens qu'un autre groupe lui a donné). Ainsi les verbes pondre et traire sont utilisés dans la langue générale avec les sens acquis dans le domaine agricole et la perte des sens étymologiques, très généraux " poser » et « tirer ». Mais, qu'il s'agisse d'extensions ou restrictions d'emploi, d'emprunts sociaux ou de véritables néologismes qui se diffusent, le résultat est le même : des mots ont changé de sens et ces sens se sont lexicalisés coexistant avec les sens originels ou provoquant leur disparition. Les exemples du début de ce paragraphe relèvent de la néologie sémantique par figure.

\subsubsection{DE LA LANGUE GÉNÉRALE À DES DOMAINES DE SPÉCIALITÉ}

Inversement, la langue générale fournit aussi des termes dans des domaines spécialisés, comme chaussette dans la physique nucléaire, jupe en mécanique ou carrosserie, etc. La langue familière a également donné des termes argotiques comme le montre le cas de biroute 'manche à air' (1916) deux ans seulement après son apparition dans l'argot, sans doute militaire, avec le sens de 'pénis' en 1914. Par ailleurs, pour revenir au domaine du sport, nombre de disciplines ou épreuves sportives sont dénommées par des mots de la langue courante, au moins comme têtes de composés ou de synapsies : saut (en hauteur, en longueur, à la perche, triple saut), lancer ( $d u$ disque, du javelot, du poids...). La souris informatique est clairement un emploi métaphorique calqué sur la métaphore anglaise mouse

\footnotetext{
${ }^{12}$ Mais faire du huit mégabits, fabriqué selon le même principe, n'est pas lexicographié.
} 
qui, de l'animal, sert aussi à dénommer le boîtier relié à un ordinateur. Puis cette souris du domaine informatique s'est vite répandue dans la langue courante, par banalisation cette fois, avec l'essor de la micro-informatique.

Parfois c'est entre trois unités que se nouent des rapports complexes : un emprunt, une recommandation officielle (souvent indiquée comme telle dans les dictionnaires) et des dénominations courantes. Ainsi phishing a-t-il comme recommandation officielle française filoutage, alors que la dénomination d'origine québécoise hameçonnage (mise en deuxième place dans les recommandations officielles françaises) s'emploie plus couramment. Le J.O. du 28.07.2013 recommande officiellement beuverie express pour binge drinking alors que circulent depuis plusieurs années déjà biture express et surtout cuite express. Les pages indiquées par Google pour beuverie express datent pour la plupart du 29.07.2013 ou d'une date postérieure.

Si presque tous les exemples donnés dans cette section sont largement répandus, utilisés par le commun des mortels quand il s'agit de domaines spécialisés auxquels ont accès de grandes franges de la population comme le domaine du sport, il n'en va de même quand il s'agit de domaines très spécialisés auxquels n'ont accès que les spécialistes du domaine. C'est le cas de la jupe de la mécanique et à plus forte raison de la chaussette de la physique nucléaire. C'est aussi le cas de l'acception « création lexicale de malades mentaux, souvent incompréhensible » de néologisme. Cet emploi daté du début du $\mathrm{XX}^{\mathrm{e}}$ siècle a le défaut de redonner l'aspect négatif que le mot néologisme avait lors de sa création au XVIII" siècle (" abus de la création et de l'usage de mots nouveaux »), et dont il a mis beaucoup de temps à se défaire. Ce mot illustre bien les va-et-vient entre registres différents, puisque, terme de critique littéraire au départ, il devient terme linguistique et pénètre dans la langue générale où il est usité, puis il ajoute un nouvel emploi qui s'ajoute aux autres qu'il détrône d'autant moins qu'il reste cantonné dans le domaine de la psychiatrie. C'est d'ailleurs la consultation des dictionnaires qui a fait découvrir à l'auteur de ces lignes cette acception qu'il ignorait complètement.

\section{Conclusion}

Les dictionnaires sont autant des outils permettant d'étudier les mots - et les versions électroniques des dictionnaires permettent de nombreuses recherches sur les dates d'apparition des mots dont on peut ensuite étudier la trajectoire - que des objets d'études pour voir comment ils définissent la néologie et les néologismes et comment ils les intègrent. Certains dictionnaires les insèrent largement (Le grand 
Larousse en son temps, Hachette maintenant, en particulier avec son supplément mots nouveaux) alors que d'autres s'y montrent réticents, comme des dictionnaires à destination des scolaires ou certaines éditions du dictionnaire de l'Académie française. Sur ce point v. Christophe Rey (2014) ainsi que Sablayrolles (2013).

Encore deux remarques métalexicographiques pour finir et conclure définitivement cette présentation. Alors que les marques d'usage se développent, on remarque l'abandon de la marque « néol. » au profit de la datation, précise ou approximative de plus en plus, et cette évolution prend bien en compte le fait que la durée d'un néologisme est variable. Du coup, c'est une autre marque qui s'est progressivement introduite dans certains dictionnaires, le Robert en particulier, indiquant la date de début de circulation si elle diffère beaucoup de la date de première attestation ${ }^{13}$.

Mais, et c'est l'ultime remarque, on ne peut que regretter les silences fréquents des dictionnaires pour l'indication des dates des nouveaux emplois, lors d'un changement de domaines ou d'échanges entre langue commune et emplois spécialisés, ainsi que l'insuffisance fréquente également de l'explicitation des mécanismes sémantiques en jeu dans ces changements et échanges (v. Sablayrolles, 2011). Mais les dictionnaires ne sont pas faits prioritairement pour les linguistes et lexicologues : c'est le vaste public des usagers de la langue qui est visé et celui-ci n'a pas les mêmes besoins, et trouvent assez largement de quoi répondre à leurs questions et satisfaire leur curiosité.

\section{Références bibliographiques}

ARRIVÉ, Michel (2009), «Un néologisme pris sur le vif: la bravitude », Neologica, $\mathrm{n}^{\circ}$ 3, Classiques Garnier, pp. 67-77.

BRÉAL, Michel (2005 [1897]), Essai de sémantique, Limoges, Lambert-Lucas.

ELCHACAR, Mireille, MARTINEZ, Camille (2008), « Une banque de données en ligne pour suivre l'évolution des nomenclatures du Petit Robert et du Petit Larousse, et leur ouverture aux mots du Québec ", http://www.pages.usherbrooke.ca/clsl/vol2no1/elchacar_martinez_vol2no1_2008. html (dernière consultation : le 01.12.2017).

GALISSON, Robert (1978), Recherches de lexicologie descriptive, la banalisation lexicale : Le vocabulaire du football dans la langue sportive, Paris, Nathan.

GARDES-TAMINE, Joëlle (1990), La Grammaire, t. 1 (Phonologie, morphologie, lexicologie), Paris, Armand Colin.

GÉVAUDAN, Paul, KOCH, Peter (2010), " Sémantique cognitive et changement lexical », in Grandes voies et chemins de traverse de la sémantique cognitive (J. François éd.), Leuven, Peeters, pp. 103-145.

${ }^{13} \mathrm{Il}$ est curieux de noter que des dictionnaires sur internet renouent avec l'ancien usage avec l'utilisation de néologisme comme marque d'usage. 
HUMBLEY, John (2000), «Évolutions du lexique » et « La Terminologie », in Histoire de la langue française 1945-2000 (G. Antoine, B. Cerquiglini dir.), Paris, CNRS Éditions, pp. 71-106 et pp. 315-338.

MARTINEZ, Camille (2013), " La mise à jour des dictionnaires : une nécessaire réévaluation du texte lexicographique », Études romanes de Brno, n 34-1, pp. 41-53.

MEILLET, Antoine (1921 et 1982 [1905/1906]), "Comment les mots changent de sens », L'année sociologique 1905/1906, repris dans Linguistique historique et linguistique générale, Paris, Champion.

NYCKEES, Vincent (1988), La sémantique, Paris, Belin.

PETIT, Gérard (2009), La dénomination : approches lexicologique et terminologique, Bibliothèque de l'Information Grammaticale 65, Leuven, Peeters.

REY, Christophe (2014), « Le traitement de la néologie dans la neuvième édition du Dictionnaire de l'Académie française », Neologica, $\mathrm{n}^{\circ}$ 8, Classiques Garnier, pp. 27-44.

SABLAYROLLES, Jean-François (2005), « Mots du sport, emprunts et langue courante », in Écrire le sport (P. Baudorre, M. Boucharenc, M. Brousse, dir.), Bordeaux, Presses Universitaires de Bordeaux, pp. 39-51.

SABLAYROLLES, Jean-François (2008), « Néologie et dictionnaire(s) comme corpus d'exclusion », in Néologie et terminologie dans les dictionnaires (J.-F. Sablayrolles éd.), Paris, H. Champion, pp. 19-36.

SABLAYROLLES, Jean-François (2010), « Archaïsme : un concept mal défini et des utilisations littéraires contrastées », in Stylistique de l'archaïsme (L. Himy-Pieri, S. Macé éds), Bordeaux, Presses Universitaires de Bordeaux, pp. 43-65.

SABLAYROLLES, Jean-François (2011), «Alain Rey, le linguiste et les mots nouveaux », in Alain Rey, vocabuliste français (F. Gaudin éd.), Limoges, Lambert-Lucas, pp. 87-98.

SABLAYROLLES, Jean-François (2013) " Le militantisme néologique au XIX siècle », in $L a$ lexicographie militante (F. Gaudin éd.), Paris, H. Champion, pp. 323-340.

\section{Dictionnaires}

Dictionnaire de la langue française Lexis (1992 [1979]), dir. J. Dubois, Paris, Larousse.

Dictionnaire Hachette (2009), dir. J.-P. Mével, Paris, Hachette.

Le nouveau Littré (2006), dir. éditorial Claude Blum, Paris, Classiques Garnier.

Le nouveau Petit Robert de la langue française (2010), de P. Robert, texte remanié et amplifié sous la direction de J. Rey-Debove et A. Rey, Paris, Le Robert.

Le petit Larousse illustré (2008), Paris, Larousse.

\section{Sitographie}

www.books.google.fr/books?isbn=2354253532.

www.fr.wikipedia.org/wiki/Glossaire_de_la_boxe\#R.

www.synec-doc.be/escrime/dico/dico_escrime-R.html\#Heading451. 